disease or functional impairment of the central nervous system, or by disturbed experience or relationships.

Elements of affect and its disturbance can be studied biologically because we share common evolutionary affective substrates with lower animals, including the basic emotions. ${ }^{3}$ However, as we move from basic to higher emotions closer attention needs to be paid to social, cultural and psychological factors. Higher emotions depend on basic emotions. But higher emotions, in turn, influence basic emotions through the impact of 'meaning'.

Meaning cannot be reduced to molecules. Different levels and models of explanation are required - a green piece of paper is not a dollar bill until social convention decrees so. Those engaged in its physical production and counterfeit surveillance need to understand the physical structure of the dollar but its major significance can only be understood at the level of meaning and social convention. Craddock et al's assertion that 'any psychological explanation is, in principle, capable of being understood at the level of cellular function' is embarrassingly naive for psychiatrists. Together with their statement that 'psychology can be considered as a sub-branch of biology, in the same way that chemistry is a sub-branch of physics' it is plainly wrong.

Psychiatry requires a broad understanding of human evolution. Behaviour narrowly defined by cellular biology is not sufficient. ${ }^{3}$ Biology, ethology and paleoanthropology have shown that social living has been the most important recent evolutionary pressure for brain development. Emotions are the glue of social interactions; from the moment of birth we are instinctually driven to engage with others. The representation of affect states in self and other (mentalisation) is vital to affect regulation and effective social adaptation. Affect regulation and mentalisation are acquired through secure attachment relationships and contribute to emotional resilience, which help us to weather the challenges that life presents us with reduced risk of psychiatric illness.

The distinguished American developmental psychologist, Jerome Kagan, writes: 'The influence of biology on human psychological functions is extensive but not unlimited. Evolutionary psychologists like to write that genes keep cultures "on a leash". However culture, like a large powerful dog, can pull the person holding the leash to new, unplanned directions' (p. 81). ${ }^{4} \mathrm{He}$ also writes: 'For reasons that are not obvious, British psychiatrists retain an interest in the psychological and sociological correlates of mental illness and have resisted a narrow biological perspective more effectively than their American counterparts' (p. 53). ${ }^{4}$ Craddock et al's formulation threatens to take British psychiatry down the American cul-de-sac. Its reductionist outlook necessarily downgrades the importance of training and continuing professional development of psychiatrists in psychological, social and cultural matters to second place.

The history of the National Health Service has been fundamental in shaping British psychiatry today; it is an example of fundamental cultural influence. The relevance of psychosocial factors to the full range of mental disorders is well documented. ${ }^{5}$ Psychiatry is a broad integrative specialty, with significant diversity within. Our core expertise is the management and not the elimination of the necessary tension between advances in biomedical science and a broader biopsychosocial model of practice. The broad biopsychosocial model is the only one consistent with the facts and not a compromise. A broad evolutionary perspective permits the examination of religion and spirituality as well as culture and society and their relevance to psychiatry.

In the context of the credit crunch British psychiatrists face renewed threats to add to old woes. Its enemies would like to caricature the specialty as limited and reductionist. Craddock et al give them further ammunition! However, Nesse ${ }^{3}$ aptly captures the core expertise of psychiatrists when he writes (in this case specifically in relation to depression): 'The clinical challenge is the same as it has always been - trying to understand people and their relationships, goals and feelings in order to understand, and help them understand, why they do what they do and why they feel what they feel. That, in combination with new diagnostic tests, genomic findings, and effective new drugs that block depression, will offer a bright future for treating depression' (pp. 171-2).

The academic and professional leadership of psychiatrists should reflect the full range of our expertise.

1 Craddock N, Kerr M, Thapar A. What is the core expertise of the psychiatrist? Psychiatrist 2010; 34: 457-60.

2 Ikkos G, Bouras N, McQueen D, St John-Smith P. Medicine, affect and mental health services. World Psychiatry 2010; 9: 35-6.

3 Nesse R. Evolutionary explanations for mood and mood disorders. In The American Psychiatric Publishing Textbook of Mood Disorders (eds DJ Stein, DJ Kupfer, AF Schatzberg). American Psychiatric Publishing, 2006.

4 Kagan J. The Three Cultures: Natural Sciences, Social Sciences and the Humanities in the 21st Century. Cambridge University Press.

5 Ikkos G. The futures of psychiatrists: external and internal challenges. Int Psychiatry 2010; 7: 79-81.

George Ikkos is director of medical education, Barnet, Enfield and Haringey Mental Health NHS Trust, consultant psychiatrist in liaison psychiatry, Royal National Orthopaedic Hospital, and honorary visiting research professor, London South Bank University, email: ikkos@doctors.org.uk; Paul St. John-Smith is consultant community psychiatrist, Hertfordshire Partnership Foundation NHS Trust (Mental Health), Watford; Daniel McQueen is consultant psychiatrist in child and adolescent psychiatry, Barnet, Enfield and Haringey Mental Health NHS Trust, London.

doi: 10.1192/pb.35.1.33a

\section{Don't forget offenders with an intellectual disability}

Appleby's editorial ${ }^{1}$ on how best to tackle offender health, particularly mental ill health and substance misuse, was a stimulating read. However, it contained no reference to the estimated 5800 prisoners with an intellectual disability. This group of offenders are effectively excluded from interventions within prisons aimed at reducing re-offending. Moreover, research from the Prison Reform Trust indicates that individuals with an intellectual disability in prison are subject to routine human rights abuses, are five times more likely to be restrained and three times more likely to be segregated compared with prisoners without intellectual disability. ${ }^{2}$ These are harrowing statistics.

Appleby highlighted three essential service provision aims for offenders with mental ill health - early intervention, alternatives to prison and multi-agency packages when leaving prison. Any services and interventions established to meet these aims for individuals with mental illness would not necessarily meet the needs of offenders with an intellectual 
disability. It is essential that thought is given to the means of providing appropriate support for them.

Throughout British psychiatric and mental health history from the closing of the Victorian asylums to community care and revisions to the Mental Health Act - policy has always been weighted more towards people with mental health problems than those with an intellectual disability. We should redress this imbalance.

Since Lord Bradley's report, ${ }^{3}$ important steps have been taken to try to improve away-from-prison diversion schemes for offenders with an intellectual disability. These include piloting a screening questionnaire for intellectual disability, due to be available in prisons next year. It is vital that the government continues to support the development of strategies for offenders with an intellectual disability as well as those with mental health problems. Budgetary restrictions should not be allowed to impede this work.

Many people appear unaware of the role of the independent sector in providing care and treatment for an increasing proportion of this patient group. In 1998, 15\% of individuals with an intellectual disability were detained within hospitals in the independent sector, but in 2008 this had grown to $46 \% .{ }^{4}$ Moreover, in 2007/2008 a total of 67 Section 37 Hospital Orders were made (restricted and unrestricted), of which 42 individuals were placed within the independent sector. $^{4}$

Collectively, the independent sector has immense expertise in how best to provide tailor-made and specialist care to this patient group. However, at a strategic level it is only consulted at a superficial level regarding the future development of services to meet the needs of this patient group. This may reflect a guardedness in relation to the commercial foundations of the independent sector, which may be linked to a misunderstanding of its funding basis. In regard to the provision of services to patients with an intellectual disability, all care is funded by the National Health Service (NHS) or Social Services, not by the individual receiving care. In effect, the independent sector works in clinical partnership with the NHS as its agency to develop and provide such care. Given this level of cooperation and the expertise of the independent sector, it seems surprising that the input from this sector is missing from the strategic development of services for this very vulnerable group of individuals.

1 Appleby L. Offender health: the next frontier. Psychiatrist 2010; 34: 409-10.

2 Prison Reform Trust. Bromley Briefings: Prison Factfile. Prison Reform Trust, 2009.

3 Lord Bradley. Lord Bradley's Review of People with Mental Health Problems or Learning Disabilities in the Criminal Justice System. Department of Health, 2009.

4 Mental Health Act Commission. Coercion and Consent: Monitoring the Mental Health Act 2007-2009 (The Mental Health Act Commission Thirteenth Biennial Report 2007-2009). TSO (The Stationery Office), 2009 .

Claire Royston is medical director, Care Principles, Newmarket, Suffolk, email: claire.royston@careprinciples.com

doi: 10.1192/pb.35.1.34 